\title{
Novel Coronavirus Disease (COVID-19) and Biologic Therapy in Psoriasis: Infection Risk and Patient Counseling in Uncertain Times
}

\author{
Nicholas D. Brownstone - Quinn G. Thibodeaux • Vidhatha D. Reddy • \\ Bridget A. Myers · Stephanie Y. Chan · Tina Bhutani • \\ Wilson Liao
}

Received: March 25, 2020 / Published online: April 16, 2020

(C) The Author(s) 2020

\begin{abstract}
With the emergence of the novel coronavirus disease (COVID-19) viral pandemic, there is uncertainty whether biologic agents for psoriasis may place patients at a higher risk for infection or more severe disease course. This commentary offers patient counseling recommendations based on the current available evidence. While there are currently no specific data for psoriasis biologics and COVID-19, data are presented here from phase III clinical trials of psoriasis biologics on rates of upper respiratory infection, influenza, and serious infection. Overall these data reveal that on the whole, psoriasis biologics do not show major increases in infection risk compared to placebo during
\end{abstract}

the course of these trials. However, as the COVID-19 virus is a novel pathogen that is associated with mortality in a subset of patients, a cautious approach is warranted. We discuss factors that may alter the benefit-risk ratio of biologic use during this time of COVID-19 outbreak. Ultimately, treatment decisions should be made on the basis of dialogue between patient and provider, considering each patient's individualized situation. Once this pandemic has passed, it is only a matter of time before a new viral disease reignites the same issues discussed here.

Keywords: Biologics; Coronavirus; COVID-19; Infection; Pandemic; Psoriasis; SARS-CoV-2

Digital Features To view digital features for this article go to https://doi.org/10.6084/m9.figshare.12063624.

N. D. Brownstone - Q. G. Thibodeaux ·

V. D. Reddy - B. A. Myers · S. Y. Chan · T. Bhutani .

W. Liao $(\square)$

Department of Dermatology, Psoriasis and Skin

Treatment Center, University of California San

Francisco, San Francisco, CA, USA

e-mail: wilson.liao@ucsf.edu 


\section{Key Summary Points}

With the emergence of the COVID-19 viral pandemic, there is uncertainty whether biologic agents for psoriasis may place patients at a higher risk for infection or worsened disease course.

While there are currently no specific data for psoriasis biologics and COVID-19, data are presented here from phase III clinical trials of psoriasis biologics on rates of upper respiratory infection, influenza, and serious infection.

Factors that should be considered when deciding whether to start or continue biologics include severity of underlying psoriasis or psoriatic arthritis; COVID-19 risk factors such as older age, cardiovascular disease, hypertension, lung disease, diabetes, or cancer; concomitant immunosuppressive medications or conditions; and risk of exposure to the COVID-19 virus based on geography, occupation, and living situation.

Ultimately, treatment decisions should be made on an individualized basis based on dialogue between patient and provider.

\section{COMMENTARY}

In late December 2019, the world was introduced to the novel coronavirus disease (COVID19). As of March 2020, there were over 920,000 cases and 46,000 deaths worldwide, with these numbers expected to rise sharply. Upon review of 55,924 patients with COVID-19 [1], the clinical presentation generally involved fever in $87.9 \%$, dry cough in $67.7 \%$, fatigue in $38.1 \%$, sputum production in $33.4 \%$, shortness of breath in $18.6 \%$, and sore throat in $13.9 \%$. Gastrointestinal symptoms have also been reported with diarrhea in $3.7 \%$ of patients and nausea or vomiting in $5 \%$ of patients. Patients with COVID-19 generally develop signs and symptoms on average 5-6 days after infection (range 1-14 days) [1]. The goal of this article is to review the known clinical trial data on infection risk with biologic therapy for psoriasis and offer patient counseling recommendations based on the current available evidence.

There are currently 11 biologic therapies approved for psoriasis. These medications are engineered to target individual mediators of inflammation including tumor necrosis factoralpha (TNF $\alpha$ ), interleukin-17 (IL-17), and IL-23. Although the safety profiles of these biologic agents are generally preferable to those of traditional immunosuppressive therapies, there is concern that treatment with these agents may reduce resistance to infection. This concern is heightened during novel disease outbreaks and the resulting increased media coverage. As a result, patients are increasingly turning to healthcare providers for guidance regarding the use of biologic agents during disease outbreaks.

Given the novel nature and rapidly evolving knowledge of COVID-19, there are currently no specific data for how biologic therapy affects patients' risk of acquiring this coronavirus infection or COVID-19 outcomes. However, more general data on infection risk are available for psoriasis biologic agents from phase III clinical trial data. Infections typically reported in these studies include upper respiratory infections, influenza, sinusitis, urinary tract infection, opportunistic infections, and serious infections. Serious infections are defined as infections involving various organ systems that may lead to hospitalization or death including pneumonia, septic arthritis, erysipelas, cellulitis, diverticulitis, pyelonephritis, and prosthetic or post-surgical infection. Table 1 summarizes the rates of upper respiratory infections, rates of influenza, and risks of serious infections for all 11 currently approved biologic agents for psoriasis, as observed in phase III clinical trials. Overall these data reveal that on the whole, psoriasis biologics do not show substantial increases in infection risk compared to placebo, which is also consistent with long-term registry data [2]. The safety signal appears especially clean for etanercept, ustekinumab, tildrakizumab, guselkumab, and risankizumab, 
Table 1 Rates of upper respiratory infection, influenza, and serious infection in the phase III clinical trials of US FDAapproved biologics for psoriasis

\begin{tabular}{|c|c|c|c|}
\hline $\begin{array}{l}\text { Medication, year } \\
\text { of FDA approval, } \\
\text { mechanism of } \\
\text { action }\end{array}$ & Rates of URI (vs. placebo) & $\begin{array}{l}\text { Rates of influenza (vs. } \\
\text { placebo) }\end{array}$ & $\begin{array}{l}\text { Rates of serious infections } \\
\text { (vs. placebo) }\end{array}$ \\
\hline \multirow{4}{*}{$\begin{array}{l}\text { Adalimumab, } \\
\text { 2002, TNFa } \\
\text { inhibitor }\end{array}$} & REVEAL [7] & REVEAL & REVEAL \\
\hline & $\begin{array}{l}7.2 \%(40 \mathrm{mg}) \text { vs. } 3.5 \% \text { (placebo) at } \\
\text { week } 16\end{array}$ & NR & $\begin{array}{l}0.6 \%(40 \mathrm{mg}) \text { vs. } 1 \% \\
\quad \text { (placebo) at week } 16\end{array}$ \\
\hline & CHAMPION [8] & CHAMPION & CHAMPION \\
\hline & $\begin{array}{l}28 \%(40 \mathrm{mg}) \text { vs. } 20.8 \% \text { (placebo) at } \\
\text { week } 16 \text { for nasopharyngitis }\end{array}$ & $\begin{array}{l}0 \%(40 \mathrm{mg}) \text { vs. } 1.9 \% \text { (placebo) } \\
\text { at week } 16 \text { for viral infection }\end{array}$ & $\begin{array}{l}0 \%(40 \mathrm{mg}) \text { vs. } 0 \% \\
\quad \text { (placebo) at week } 16\end{array}$ \\
\hline \multirow{6}{*}{$\begin{array}{l}\text { Etanercept, } 2004 \\
\text { TNFa inhibitor }\end{array}$} & Tyring et. al. [9] & Tyring et. al. [9] & Tyring et. al. [9] \\
\hline & $\begin{array}{l}\text { 20.2/100 PY }(50 \mathrm{mg} \text { ) vs. } 24.3 / 100 \mathrm{PY} \\
\text { (placebo) through week } 96\end{array}$ & NR & $\begin{array}{l}1.2 / 100 \mathrm{PY}(50 \mathrm{mg}) \text { vs. } 1.5 / \\
100 \mathrm{PY} \text { (placebo) through } \\
\text { week } 96\end{array}$ \\
\hline & Papp et al. $[10]$ & Papp et al. $[10]$ & Papp et al. $[10]$ \\
\hline & $\begin{array}{l}13 \%(50 \mathrm{mg}) \text { vs. } 13 \%(25 \mathrm{mg}) \text { vs. } 13 \% \\
\quad \text { (placebo) at week } 12\end{array}$ & $\begin{array}{l}4 \% \text { (50 mg) vs. } 5 \%(25 \mathrm{mg}) \text { vs. } \\
2 \% \text { (placebo) at week } 12 \text { for } \\
\text { "flu syndrome" }\end{array}$ & $\begin{array}{l}\mathrm{NR}(<5 \% \text { reported in } \\
\text { study })\end{array}$ \\
\hline & Leonardi et. al. [11] & Leonardi et. al. [11] & Leonardi et. al. [11] \\
\hline & $\begin{array}{l}\text { 5\% (50 mg BIW) vs. } 9 \%(25 \mathrm{mg} \\
\text { BIW) vs. } 10 \%(25 \mathrm{mg} \text { QWK) vs. } \\
11 \% \text { (placebo) at week } 12\end{array}$ & $\mathrm{NR}(<5 \%$ reported in study $)$ & $\begin{array}{l}\mathrm{NR}(<5 \% \text { reported in } \\
\text { study })\end{array}$ \\
\hline \multirow{4}{*}{$\begin{array}{l}\text { Infliximab, 2006, } \\
\text { TNF } \alpha \text { inhibitor }\end{array}$} & EXPRESS $1[12]$ & EXPRESS 1 & EXPRESS 1 \\
\hline & $\begin{array}{l}15 \%(5 \mathrm{mg} / \mathrm{kg}) \text { vs. } 16 \% \text { (placebo) at } \\
\text { week } 24\end{array}$ & NR & NR \\
\hline & EXPRESS 2 [13] & EXPRESS 2 & EXPRESS 2 \\
\hline & $\begin{array}{c}16 \%(3 \mathrm{mg} / \mathrm{kg}) \text { vs. } 13.4 \%(5 \mathrm{mg} / \mathrm{kg}) \\
\text { vs. } 14 \% \text { (placebo) at week } 14\end{array}$ & NR & NR \\
\hline
\end{tabular}


Table 1 continued

\begin{tabular}{|c|c|c|c|}
\hline $\begin{array}{l}\text { Medication, year } \\
\text { of FDA approval, } \\
\text { mechanism of } \\
\text { action }\end{array}$ & Rates of URI (vs. placebo) & $\begin{array}{l}\text { Rates of influenza (vs. } \\
\text { placebo) }\end{array}$ & $\begin{array}{l}\text { Rates of serious infections } \\
\text { (vs. placebo) }\end{array}$ \\
\hline \multirow{6}{*}{$\begin{array}{l}\text { Certolizumab, } \\
\text { 2018, } \\
\text { PEGylated } \\
\text { TNF } \alpha \text { inhibitor }\end{array}$} & CIMPASI 1 [14] & CIMPASI 1 & CIMPASI 1 \\
\hline & $\begin{array}{l}9.1 \%(400 \mathrm{mg}) \text { vs. } 7.4 \%(200 \mathrm{mg}) \text { vs. } \\
\quad 5.9 \% \text { (placebo) at week } 16\end{array}$ & NR & $\begin{array}{l}0 \%(400 \mathrm{mg}) \text { vs. } 0 \% \\
(200 \mathrm{mg}) \text { vs. } 0 \% \text { (placebo) } \\
\text { at week } 16\end{array}$ \\
\hline & CIMPASI 2 [14] & CIMPASI 2 & CIMPASI 2 \\
\hline & $\begin{array}{l}5.7 \% \text { ( } 400 \mathrm{mg} \text { ) vs. } 4.4 \%(200 \mathrm{mg}) \text { vs. } \\
4.1 \% \text { (placebo) at week } 16\end{array}$ & NR & $\begin{array}{l}1.1 \%(400 \mathrm{mg}) \text { vs. } 0 \% \\
(200 \mathrm{mg}) \text { vs. } 0 \% \text { (placebo) } \\
\text { at week } 16\end{array}$ \\
\hline & CIMPACT [28] & CIMPACT & CIMPACT \\
\hline & $\begin{array}{l}3.6 \%(200 \mathrm{mg}) \text { vs. } 10.5 \% \text { (placebo) at } \\
\text { week } 12\end{array}$ & NR & $0 \%$ vs. $0 \%$ at week 12 \\
\hline \multirow{4}{*}{$\begin{array}{l}\text { Ustekinumab, } \\
\text { 2009, anti-IL- } \\
\text { 12/23 }\end{array}$} & PHOENIX $1[15]$ & PHOENIX 1 & PHOENIX 1 \\
\hline & $\begin{array}{c}7.1 \%(45 \mathrm{mg}) \text { vs. } 6.3 \%(90 \mathrm{mg}) \text { vs. } \\
6.3 \% \text { (placebo) at week } 12\end{array}$ & NR & $\begin{array}{l}0 \%(45 \mathrm{mg}) \text { vs. } 0.8 \% \\
\quad(90 \mathrm{mg}) \text { vs. } 0.4 \% \\
\text { (placebo) at week } 12\end{array}$ \\
\hline & PHOENIX 2 [16] & PHOENIX 2 & PHOENIX 2 \\
\hline & $\begin{array}{l}4.4 \% \text { ( } 45 \mathrm{mg} \text { ) vs. } 2.9 \%(90 \mathrm{mg} \text { ) vs. } \\
3.4 \% \text { (placebo) at week } 12\end{array}$ & NR & $\begin{array}{l}0 \% \text { ( } 45 \mathrm{mg} \text { ) vs. } 0.2 \% \\
\quad(90 \mathrm{mg} \text { ) vs. } 0.5 \% \\
\text { (placebo) at week } 12\end{array}$ \\
\hline
\end{tabular}


Table 1 continued

\begin{tabular}{|c|c|c|c|}
\hline $\begin{array}{l}\text { Medication, year } \\
\text { of FDA approval, } \\
\text { mechanism of } \\
\text { action }\end{array}$ & Rates of URI (vs. placebo) & $\begin{array}{l}\text { Rates of influenza (vs. } \\
\text { placebo) }\end{array}$ & $\begin{array}{l}\text { Rates of serious infections } \\
\text { (vs. placebo) }\end{array}$ \\
\hline \multirow{8}{*}{$\begin{array}{l}\text { Secukinumab, } \\
\text { 2015, anti-IL- } \\
\text { 17A }\end{array}$} & ERASURE [17] & ERASURE & ERASURE \\
\hline & $\begin{array}{l}3.7 \%(300 \mathrm{mg} \text { ) vs. } 4.1 \%(150 \mathrm{mg}) 0 \% \\
\quad \text { (placebo) at week } 12\end{array}$ & $\begin{array}{l}2 \%(300 \mathrm{mg}) \text { vs. } 1.2 \% \\
\quad(150 \mathrm{mg}) \text { vs. } 1.2 \% \text { (placebo) } \\
\text { at week } 12\end{array}$ & $\begin{array}{l}1 \%(300 \mathrm{mg}) \text { vs. } 0.7 \% \\
\quad(150 \mathrm{mg}) \text { vs. } 1.5 \% \\
\text { (placebo) at week } 52\end{array}$ \\
\hline & FIXTURE [17] & & FIXTURE \\
\hline & $\begin{array}{l}2.1 \%(300 \mathrm{mg}) \text { vs. } 3.1 \%(150 \mathrm{mg}) \text { vs. } \\
0.9 \% \text { (placebo) at week } 12\end{array}$ & & $\begin{array}{l}1.1 \%(300 \mathrm{mg}) \text { vs. } 0.6 \% \\
(150 \mathrm{mg}) \text { vs. } 0.3 \% \\
\text { (placebo) at week } 52\end{array}$ \\
\hline & FEATURE [18] & & \\
\hline & $\begin{array}{l}5.1 \%(300 \mathrm{mg}) \text { vs. } 5.1 \%(150 \mathrm{mg}) \text { vs. } \\
8.5 \% \text { (placebo) at week } 12 \text { for } \\
\text { nasopharyngitis }\end{array}$ & & \\
\hline & JUNCTURE [19] & & \\
\hline & $\begin{array}{l}\text { Sinusitis: } 5 \% \text { ( } 300 \mathrm{mg} \text { ) vs. } 1.6 \% \\
\text { (150 mg) vs. } 0 \% \text { (placebo); } \\
\text { Nasopharyngitis: } 31.7 \%(300 \mathrm{mg}) \\
\text { vs. } 23 \%(150 \mathrm{mg} \text { ) vs. } 16.4 \% \\
\text { (placebo) at week } 12\end{array}$ & & \\
\hline \multirow{6}{*}{$\begin{array}{l}\text { Brodalumab, } \\
\text { 2017, anti-IL-17 }\end{array}$} & AMAGINE 1 [20] & AMAGINE 1 & AMAGINE 1 \\
\hline & $\begin{array}{l}\text { 8.2\% (140 mg Q2W) vs. 8.1\% } \\
(210 \mathrm{mg} \text { Q2W) vs. } 6.4 \% \text { (placebo) }\end{array}$ & NR & $\begin{array}{l}0.9 \%(140 \mathrm{mg} \text { Q2W) vs. } \\
0.5 \%(210 \mathrm{mg} \mathrm{Q} 2 \mathrm{~W}) \text { vs. } \\
0 \% \text { (placebo) at week } 12 \\
4.5 \%(140 \mathrm{mg} \mathrm{Q} 2 \mathrm{~W}) \text { vs. } \\
1.2 \%(210 \mathrm{mg} \mathrm{Q} 2 \mathrm{~W}) \text { at } \\
\text { week } 52\end{array}$ \\
\hline & AMAGINE 2 [21] & AMAGINE 2 & AMAGINE 2 \\
\hline & NR & NR & NR \\
\hline & AMAGINE 3 [21] & AMAGINE 3 & AMAGINE 3 \\
\hline & NR & NR & NR \\
\hline
\end{tabular}


Table 1 continued

\begin{tabular}{|c|c|c|c|}
\hline $\begin{array}{l}\text { Medication, year } \\
\text { of FDA approval, } \\
\text { mechanism of } \\
\text { action }\end{array}$ & Rates of URI (vs. placebo) & $\begin{array}{l}\text { Rates of influenza (vs. } \\
\text { placebo) }\end{array}$ & $\begin{array}{l}\text { Rates of serious infections } \\
\text { (vs. placebo) }\end{array}$ \\
\hline \multirow[t]{3}{*}{$\begin{array}{l}\text { Ixekizumab, 2017, } \\
\text { anti-IL-17A }\end{array}$} & UNCOVER 1, 2, 3 (pooled) [22] & UNCOVER 1, 2, 3 (pooled) & $\begin{array}{l}\text { UNCOVER 1, 2, } 3 \\
\text { (pooled) }\end{array}$ \\
\hline & $\begin{array}{c}3.9 \%(\mathrm{Q} 4 \mathrm{~W}) \text { vs. } 4.4 \%(\mathrm{Q} 2 \mathrm{~W}) \text { vs. } \\
3.5 \% \text { (placebo) at week } 12\end{array}$ & NR & $\begin{array}{l}0.7 \%(\mathrm{Q} 4 \mathrm{~W}) \text { vs. } 0.4 \% \\
(\mathrm{Q} 2 \mathrm{~W}) \text { vs. } 0.4 \% \text { (placebo) } \\
\text { at week } 12\end{array}$ \\
\hline & $10 \%$ (IXE all exposure) at week 60 & & $\begin{array}{l}\text { 1.4\% (IXE all exposure) at } \\
\text { week } 60\end{array}$ \\
\hline \multirow{4}{*}{$\begin{array}{l}\text { Guselkumab, } \\
\text { 2017, anti-IL-23 }\end{array}$} & VOYAGE 1 [23] & VOYAGE 1 & VOYAGE 1 \\
\hline & $\begin{array}{l}7.6 \%(100 \mathrm{mg}) \text { vs. } 5.2 \% \text { (placebo) at } \\
\text { week } 16\end{array}$ & NR & $\begin{array}{l}0 \%(100 \mathrm{mg} \text { ) vs. } 0 \% \\
\quad \text { (placebo) at week } 16\end{array}$ \\
\hline & VOYAGE 2 [24] & VOYAGE 2 & VOYAGE 2 \\
\hline & $\begin{array}{l}3.2 \%(100 \mathrm{mg}) \text { vs. } 4.0 \% \text { (placebo) at } \\
\text { week } 16\end{array}$ & NR & $\begin{array}{l}0.2 \%(100 \mathrm{mg}) \text { vs. } 0.4 \% \\
\text { (placebo) at week } 16\end{array}$ \\
\hline \multirow{4}{*}{$\begin{array}{l}\text { Tildrakizumab, } \\
\text { 2018, anti-IL-23 }\end{array}$} & RESURFACE 1 [25] & RESURFACE 1 & RESURFACE 1 \\
\hline & $\begin{array}{l}3 \%(100 \mathrm{mg}) \text { vs. } 5 \%(200 \mathrm{mg}) \text { vs. } 6 \% \\
\text { (placebo) at week } 12\end{array}$ & NR & $\begin{array}{l}<1 \%(100 \mathrm{mg}) \text { vs. }<1 \% \\
(200 \mathrm{mg}) \text { vs. } 0 \% \text { (placebo) } \\
\text { at week } 12\end{array}$ \\
\hline & RESURFACE 2 [25] & RESURFACE 2 & RESURFACE 2 \\
\hline & $\begin{array}{l}0 \%(100 \mathrm{mg}) \text { vs. } 0 \%(200 \mathrm{mg}) \text { vs. } 0 \% \\
\quad \text { (placebo) at week } 12\end{array}$ & NR & $\begin{array}{l}0 \%(100 \mathrm{mg}) \text { vs. }<1 \% \\
(200 \mathrm{mg}) \text { vs. } 1 \% \text { (placebo) } \\
\text { at week } 12\end{array}$ \\
\hline
\end{tabular}


Table 1 continued

\begin{tabular}{|c|c|c|c|}
\hline $\begin{array}{l}\text { Medication, year } \\
\text { of FDA approval, } \\
\text { mechanism of } \\
\text { action }\end{array}$ & Rates of URI (vs. placebo) & $\begin{array}{l}\text { Rates of influenza (vs. } \\
\text { placebo) }\end{array}$ & $\begin{array}{l}\text { Rates of serious infections } \\
\text { (vs. placebo) }\end{array}$ \\
\hline \multirow{8}{*}{$\begin{array}{l}\text { Risankizumab, } \\
\text { 2019, anti-IL-23 }\end{array}$} & ULTIMMA-1 [26] & ULTIMMA-1 & ULTIMMA-1 \\
\hline & $\begin{array}{l}\text { Part A: } 5.59 \%(150 \mathrm{mg}) \text { vs. } 1.96 \% \\
\quad \text { (placebo) at week } 16\end{array}$ & $\begin{array}{l}\text { Part A: } 6.58 \%(150 \mathrm{mg}) \text { vs. } \\
5.88 \% \text { (placebo) at week } 16 \\
\text { viral upper respiratory } \\
\text { infection }\end{array}$ & $\begin{array}{l}\text { Part A: } 0.3 \%(150 \mathrm{mg}) \text { vs. } \\
0 \% \text { (placebo) at week } 16\end{array}$ \\
\hline & $\begin{array}{l}\text { Part B: } 10.10 \%(150 \mathrm{mg}) \text { vs. } 8.25 \% \\
\quad \text { (placebo) at weeks } 16-52\end{array}$ & $\begin{array}{l}\text { Part B: } 13.47 \%(150 \mathrm{mg}) \text { vs. } \\
15.46 \% \text { (placebo) at weeks } \\
\text { 16-52 for viral upper } \\
\text { respiratory infection }\end{array}$ & $\begin{array}{l}\text { Part B: } 0.7 \%(150 \mathrm{mg}) \text { vs. } \\
\text { 1\% (placebo) at weeks } \\
16-52\end{array}$ \\
\hline & ULTIMMA-2 [26] & ULTIMMA-2 & ULTIMMA-2 \\
\hline & $\begin{array}{l}\text { Part A: } 3.74 \%(150 \mathrm{mg}) \text { vs. } 2.04 \% \\
\text { (placebo) at week } 16\end{array}$ & $\begin{array}{l}\text { Part A: } 2.04 \%(150 \mathrm{mg}) 1.02 \% \\
\text { (placebo) at week } 16 \text { for } \\
\text { influenza }\end{array}$ & $\begin{array}{l}\text { Part A: } 1 \%(150 \mathrm{mg}) \text { vs. } 0 \% \\
\text { (placebo) at week } 16\end{array}$ \\
\hline & $\begin{array}{l}\text { Part B: } 8.25 \%(150 \mathrm{mg}) \text { vs. } 9.57 \% \\
\quad \text { (placebo) at weeks } 16-52\end{array}$ & $\begin{array}{l}\text { Part B: } 1.37 \%(150 \mathrm{mg}) \text { vs. } \\
2.13 \% \text { (placebo) at weeks } \\
16-52 \text { for influenza }\end{array}$ & $\begin{array}{l}\text { Part B: } 0.7 \%(150 \mathrm{mg}) \text { vs. } \\
\text { 0\% (placebo) at weeks } \\
16-52\end{array}$ \\
\hline & IMMHANCE [27] & IMMHANCE & IMMHANCE \\
\hline & $\begin{array}{l}\text { Part A1: } 1.47 \%(150 \mathrm{mg}) \text { vs. } 5 \% \\
\quad \text { (placebo) at week } 16\end{array}$ & $\begin{array}{l}\text { Part A1: } 0.74 \%(150 \mathrm{mg}) \text { vs. } \\
\text { 1\% (placebo) at week } 16\end{array}$ & $\begin{array}{l}0 \%(150 \mathrm{mg}) \text { vs. } 0 \% \\
\text { (placebo) at week } 16 \text { for } \\
\text { viral infection, bronchitis, } \\
\text { and bacterial meningitis }\end{array}$ \\
\hline
\end{tabular}

$N R$ not reported, $N S$ not significant, $P Y$ patient year

although it is important to note that the data from these trials were derived from a relatively short period and do not fully reflect real-world settings.

Biologic medications for psoriasis are meant to be taken continuously. There are risks to stopping biologic therapy since psoriasis flares and erythroderma may lead to poor quality of life and hospitalization. Also, stopping and restarting some biologic agents may result in reduced efficacy $[3,4]$. On the other hand, given the absence of specific data on psoriasis biologics and COVID-19, which can potentially be fatal, a cautious approach is warranted. In particular, the presence of risk factors for COVID-19 mortality such as age $>60$, cardiovascular disease, hypertension, lung disease, diabetes, or cancer may alter the benefit-risk ratio for biologic therapy, particularly in the short term whereby biologic reduction or discontinuation may not lead to immediate disease flare [5] (Table 2). 
Table 2 Considerations for use of psoriasis biologic medications during the COVID-19 pandemic

\begin{tabular}{|c|c|}
\hline $\begin{array}{l}\text { Factors favoring biologic discontinuation or reduction in } \\
\text { immunomodulatory regimen }\end{array}$ & Factors favoring biologic continuation \\
\hline Any active infection, including COVID-19 & Young age \\
\hline $\begin{array}{l}\text { COVID-19 risk factors including: age }>60 \text {, cardiovascular } \\
\text { disease, hypertension, lung disease, diabetes, or cancer }\end{array}$ & No COVID-19 high risk co-morbidities \\
\hline $\begin{array}{l}\text { Concomitant immunosuppression (e.g., methotrexate, } \\
\text { prednisone, cyclosporine) }\end{array}$ & Biologic monotherapy \\
\hline Immunosuppressive condition (e.g., HIV) & $\begin{array}{l}\text { Severe underlying psoriasis or psoriatic arthritis, with history } \\
\text { of rapid flares or unstable subtypes (pustular, } \\
\text { erythrodermic) }\end{array}$ \\
\hline History of infections while on biologic & No concomitant immunosuppressive conditions \\
\hline Mild-to-moderate underlying psoriasis & Low risk of exposure to COVID-19 virus \\
\hline $\begin{array}{l}\text { High risk of exposure to COVID-19 virus (e.g., endemic } \\
\text { area, healthcare worker, nursing home resident, household } \\
\text { member or co-worker with COVID-19 infection) }\end{array}$ & Long duration of COVID-19 pandemic \\
\hline Short duration of COVID-19 pandemic & \\
\hline
\end{tabular}

Therefore, at the current time, the following guidance may be given to patients with psoriasis:

1. All patients should be reminded to practice good infection prevention measures such as frequent hand washing, social distancing, and the use of telehealth resources when available.

2. There is no evidence to recommend prophylactically stopping or postponing biologic therapy in all patients with psoriasis; however, patients should have individualized discussions with their medical providers taking into account the following factors:

- COVID-19 risk factors such as older age, cardiovascular disease, hypertension, lung disease, diabetes, or cancer

- Severity of underlying psoriasis or psoriatic arthritis

- Concomitant immunosuppressive medications or conditions

- Risk of exposure to COVID-19 based on occupation or living situation
3. If a reduction in immunosuppressive treatment is desired, options include:

- Temporary discontinuation of the biologic

- Reduction in biologic dose frequency

- Transition to an alternative biologic

- Reduction or discontinuation of concomitant immunosuppressants (e.g., methotrexate)

- Increase in use of topical agents, home phototherapy, or other non-immunosuppressive medications

4. Patients who test positive for COVID-19 infection should be advised to hold their biologic dose until their infection clears. This requires resolution of fever without the use of fever-reducing medications, improvement in respiratory symptoms (e.g., cough, shortness of breath), and two negative COVID-19 test performed $24 \mathrm{~h}$ apart. However, if COVID-19 retesting is not available, then a conservative approach would be to avoid restarting biologic therapy until 30 days after resolution of fever and 
respiratory symptoms. This estimate is based on a mean duration of COVID-19 viral shedding from illness onset of 20 days (range 8-37 days) in hospitalized patients [6].

5. The risks and benefits of initiating biologic therapy should be considered on an individual patient basis, according to the factors listed above.

It is important to remember that this is a novel, rapidly changing situation, and recommendations may change as more data become available. This article is based on previously conducted studies and does not contain any studies with human participants or animals performed by any of the authors. This worldwide pandemic of substantial human disease caused by a type of virus previously thought to be relatively benign highlights the perpetual challenge of emerging infectious diseases, the importance of long-term monitoring of patients on biologic therapy, and shared decision-making with patients on biologic therapy. Once this pandemic has passed, it is only a matter of time before a new viral disease reignites the same issues discussed here.

\section{ACKNOWLEDGEMENTS}

Funding. No funding or sponsorship was received for this study or publication of this article.

Authorship. All named authors meet the International Committee of Medical Journal Editors (ICMJE) criteria for authorship for this article, take responsibility for the integrity of the work as a whole, and have given their approval for this version to be published.

Disclosures. Nicholas Brownstone, Quinn Thibodeaux, Vidhatha Reddy, Bridget Myers, and Stephanie Chan have nothing to disclose. Wilson Liao is editorial board member of the journal and is funded in part by grants from the National Institutes of Health (5U01AI119125) and Abbvie, Amgen, Janssen, Novartis,
Regeneron, Sanofi, and TRex Bio. Tina Bhutani is currently an investigator for Celgene, Janssen, Merck, and Regeneron. She has served as an advisor for Abbvie, Lilly, and Pfizer.

Compliance with Ethics Guidelines. This article is based on previously conducted studies and does not contain any studies with human participants or animals performed by any of the authors.

Data Availability. Data sharing is not applicable to this article as no datasets were generated or analyzed during the current study.

Open Access. This article is licensed under a Creative Commons Attribution-NonCommercial 4.0 International License, which permits any non-commercial use, sharing, adaptation, distribution and reproduction in any medium or format, as long as you give appropriate credit to the original author(s) and the source, provide a link to the Creative Commons licence, and indicate if changes were made. The images or other third party material in this article are included in the article's Creative Commons licence, unless indicated otherwise in a credit line to the material. If material is not included in the article's Creative Commons licence and your intended use is not permitted by statutory regulation or exceeds the permitted use, you will need to obtain permission directly from the copyright holder. To view a copy of this licence, visit http://creativecommons.org/licenses/bync/4.0/.

\section{REFERENCES}

1. World Health Organization. Report of the WHO-China Joint Mission on Coronavirus Disease 2019 (COVID-19). https://www.who.int/docs/defaultsource/coronaviruse/who-china-joint-mission-oncovid-19-final-report.pdf. Accessed Mar 21, 2020.

2. Gooderham M, Papp K. Clinical trial and registry data. Adverse React Biol. 2018;53:15-27. https:// doi.org/10.1159/000478074.

3. Reich K, Ortonne J-P, Gottlieb AB, et al. Successful treatment of moderate to severe plaque psoriasis 
with the PEGylated Fab' certolizumab pegol: results of a phase II randomized, placebo-controlled trial with a re-treatment extension. $\mathrm{Br} \mathrm{J}$ Dermatol. 2012;167(1):180-90. https://doi.org/10.1111/j. 1365-2133.2012.10941.x.

4. Ortonne J-P, Taïeb A, Ormerod AD, et al. Patients with moderate-to-severe psoriasis recapture clinical response during re-treatment with etanercept. Br J Dermatol. 2009;161(5):1190-5. https://doi.org/10. 1111/j.1365-2133.2009.09238.x.

5. Zhou F, Yu T, Du R, et al. Clinical course and risk factors for mortality of adult inpatients with COVID-19 in Wuhan, China: a retrospective cohort study. Lancet. 2020. https://doi.org/10.1016/S01406736(20)30566-3.

6. Zhou F, Yu T, Du R, et al. Clinical course and risk factors for mortality of adult inpatients with COVID-19 in Wuhan, China: a retrospective cohort study. Lancet. 2020. https://doi.org/10.1016/S01406736(20)30566-3.

7. Menter A, Tyring SK, Gordon K, et al. Adalimumab therapy for moderate to severe psoriasis: a randomized, controlled phase III trial. J Am Acad Dermatol. 2008;58(1):106-15. https://doi.org/10. 1016/j.jaad.2007.09.010.

8. Saurat J-H, Stingl G, Dubertret L, et al. Efficacy and safety results from the randomized controlled comparative study of adalimumab vs. methotrexate vs. placebo in patients with psoriasis (CHAMPION). Br J Dermatol. 2008;158(3):558-66. https://doi.org/ 10.1111/j.1365-2133.2007.08315.x.

9. Tyring S, Gordon KB, Poulin Y, et al. Long-term safety and efficacy of $50 \mathrm{mg}$ of etanercept twice weekly in patients with psoriasis. Arch Dermatol. 2007;143(6):719-26. https://doi.org/10.1001/ archderm.143.6.719.

10. Papp KA, Tyring S, Lahfa M, et al. A global phase III randomized controlled trial of etanercept in psoriasis: safety, efficacy, and effect of dose reduction. $\mathrm{Br}$ J Dermatol. 2005;152(6):1304-12. https://doi.org/ 10.1111/j.1365-2133.2005.06688.x.

11. Leonardi CL, Powers JL, Matheson RT, et al. Etanercept as monotherapy in patients with psoriasis. N Engl J Med. 2003;349(21):2014-22. https://doi. org/10.1056/NEJMoa030409.

12. Reich $\mathrm{K}$, Nestle FO, Papp $\mathrm{K}$, et al. Infliximab induction and maintenance therapy for moderateto-severe psoriasis: a phase III, multicentre, doubleblind trial. Lancet. 2005;366(9494):1367-74. https://doi.org/10.1016/S0140-6736(05)67566-6.

13. Menter MDA, Feldman SR, Weinstein GD. A randomized comparison of continuous versus intermittent infliximab maintenance regimens over 1 year in the treatment of moderate-to-severe plaque psoriasis. J Am Acad Dermatol. 2007;56:e1-e15.

14. Gottlieb AB, Blauvelt A, Thaçi D, et al. Certolizumab pegol for the treatment of chronic plaque psoriasis: results through 48 weeks from 2 phase 3, multicenter, randomized, double-blinded, placebo-controlled studies (CIMPASI-1 and CIMPASI-2). J Am Acad Dermatol. 2018;79(2):302-314. e6. https://doi.org/10.1016/j.jaad.2018.04.012.

15. Leonardi CL, Kimball AB, Papp KA, et al. Efficacy and safety of ustekinumab, a human interleukin$12 / 23$ monoclonal antibody, in patients with psoriasis: 76-week results from a randomised, doubleblind, placebo-controlled trial (PHOENIX 1). Lancet. 2008;371(9625):1665-744. https://doi.org/10. $1016 /$ S0140-6736(08)60725-4.

16. Papp KA, Langley RG, Lebwohl M, et al. Efficacy and safety of ustekinumab, a human interleukin$12 / 23$ monoclonal antibody, in patients with psoriasis: 52-week results from a randomised, doubleblind, placebo-controlled trial (PHOENIX 2). Lancet. 2008;371(9625):1675-84. https://doi.org/10. 1016/S0140-6736(08)60726-6.

17. Langley RG, Elewski BE, Lebwohl M, et al. Secukinumab in plaque psoriasis-results of two phase 3 trials. N Engl J Med. 2014;371(4):326-38. https:// doi.org/10.1056/NEJMoa1314258.

18. Blauvelt A, Prinz JC, Gottlieb AB, et al. Secukinumab administration by pre-filled syringe: efficacy, safety and usability results from a randomized controlled trial in psoriasis (FEATURE). Br J Dermatol. 2015;172(2):484-93. https://doi.org/10. 1111/bjd.13348.

19. Paul C, Lacour J-P, Tedremets L, et al. Efficacy, safety and usability of secukinumab administration by autoinjector/pen in psoriasis: a randomized, controlled trial (JUNCTURE). J Eur Acad Dermatol Venereol. 2015;29(6):1082-90. https://doi.org/10. $1111 / j d v .12751$.

20. Papp KA, Reich K, Paul C, et al. A prospective phase III, randomized, double-blind, placebo-controlled study of brodalumab in patients with moderate-tosevere plaque psoriasis. Br J Dermatol. 2016;175(2): 273-86. https://doi.org/10.1111/bjd.14493.

21. Papp KA, Gordon KB, Langley RG, et al. Impact of previous biologic use on the efficacy and safety of brodalumab and ustekinumab in patients with moderate-to-severe plaque psoriasis: integrated analysis of the randomized controlled trials AMAGINE-2 and AMAGINE-3. Br J Dermatol. 2018;179(2):320-8. https://doi.org/10.1111/bjd. 16464. 
22. Gordon KB, Blauvelt A, Papp KA, et al. Phase 3 trials of ixekizumab in moderate-to-severe plaque psoriasis. N Engl J Med. 2016;375(4):345-56. https://doi. org/10.1056/NEJMoa1512711.

23. Blauvelt A, Papp KA, Griffiths CEM, et al. Efficacy and safety of guselkumab, an anti-interleukin-23 monoclonal antibody, compared with adalimumab for the continuous treatment of patients with moderate to severe psoriasis: results from the phase III, double-blinded, placebo- and active comparator-controlled VOYAGE 1 trial. J Am Acad Dermatol. 2017;76(3):405-17. https://doi.org/10.1016/j. jaad.2016.11.041.

24. Reich K, Armstrong AW, Foley P, et al. Efficacy and safety of guselkumab, an anti-interleukin-23 monoclonal antibody, compared with adalimumab for the treatment of patients with moderate to severe psoriasis with randomized withdrawal and retreatment: results from the phase III, double-blind, placebo- and active comparator-controlled VOYAGE 2 trial. J Am Acad Dermatol. 2017;76(3):418-31. https://doi.org/10.1016/j.jaad.2016.11.042.

25. Reich K, Papp KA, Blauvelt A, et al. Tildrakizumab versus placebo or etanercept for chronic plaque psoriasis (reSURFACE 1 and reSURFACE 2): results from two randomised controlled, phase 3 trials. Lancet. 2017;390(10091):276-88. https://doi.org/ $10.1016 /$ S0140-6736(17)31279-5.

26. Gordon KB, Strober B, Lebwohl M, et al. Efficacy and safety of risankizumab in moderate-to-severe plaque psoriasis (UltIMMa-1 and UltIMMa-2): results from two double-blind, randomised, placebo-controlled and ustekinumab-controlled phase 3 trials. Lancet. 2018;392(10148):650-61. https:// doi.org/10.1016/S0140-6736(18)31713-6.

27. Langley R, Blauvelt A, Gooderham M, et al. Efficacy and safety of continuous Q12W risankizumab versus treatment withdrawal: results from the phase 3 IMMhance trial. J Am Acad Dermatol. 2019;81(4, Supplement 1):AB52. https://doi.org/10.1016/j. jaad.2019.06.220.

28. Lebwohl M, Blauvelt A, Paul C, et al. Certolizumab pegol for the treatment of chronic plaque psoriasis: Results through 48 weeks of a phase 3 , multicenter, randomized, double-blind, etanercept- and placebo-controlled study (CIMPACT). J Am Acad Dermatol. 2018;79(2):266-76.e5. https://doi.org/10. 1016/j.jaad.2018.04.013. 